\title{
Case Report \\ Herbaspirillum Infection in Humans: A Case Report and Review of Literature
}

\author{
Rashmi Dhital (D), Anish Paudel, Nidrit Bohra, and Ann K. Shin \\ Reading Hospital and Medical Center, Tower Health System, West Reading, PA, USA \\ Correspondence should be addressed to Rashmi Dhital; rashmi046@gmail.com
}

Received 5 December 2019; Revised 2 February 2020; Accepted 4 February 2020; Published 20 February 2020

Academic Editor: Raul Colodner

Copyright (C) 2020 Rashmi Dhital et al. This is an open access article distributed under the Creative Commons Attribution License, which permits unrestricted use, distribution, and reproduction in any medium, provided the original work is properly cited.

\begin{abstract}
Introduction. Herbaspirillum seropedicae are Gram-negative oxidase-positive nonfermenting rods of Betaproteobacteria class, commonly found in rhizosphere. More recently, some Herbaspirillium species have transitioned from environment to human hosts, mostly as opportunistic (pathogenic) bacteria. We present a 58-year-old female with non-small-cell lung cancer (NSCLC) who presented with pneumonia and was found to have Herbaspirillum seropedicae bacteremia. Case History. A 58-year-old woman with NSCLC on Pralsetinib presented with fevers and rigors for 2 days. Coarse breath sounds were auscultated on the right upper lung field. Labs revealed leukopenia and mild neutropenia. CT chest revealed right upper lobe pneumonia. She was admitted for sepsis secondary to pneumonia and placed on broad spectrum antibiotics with intravenous piperacillin-tazobactam and vancomycin. The patient continued to have fever 2 days after admission (max: $102.8^{\circ} \mathrm{F}$ ). Preliminary blood cultures grew Gramnegative rods. The patient continued to have temperature spikes on the 3 rd day of antibiotics $\left(T_{\max } 101.5^{\circ} \mathrm{F}\right)$. Blood cultures revealed oxidase-positive nonfermenting rods. The patient's antibiotic was changed to IV meropenem on the 4th day of hospitalization. Ultimately, on the seventh day of hospitalization, the blood culture was confirmed from outside lab as Herbaspirillum seropedicae. The patient started feeling better and defervesced after about 24 hours. Discussion. More recently, Herbaspirillum spp. have been recovered from humans. Our patient had Herbaspirillum bacteremia, and reported regularly cleaning her pond and weeding her garden with possible exposure to this environmental proteobacterium. Herbaspirillum may be more prevalent than earlier thought owing to misidentification. With the institution of appropriate antimicrobial therapy, the outcomes seem mostly favorable.
\end{abstract}

\section{Introduction}

Herbaspirillum seropedicae are Gram-negative oxidasepositive nonfermenting rods of Betaproteobacteria class, commonly found in the rhizosphere of maize and rice, which promote plant growth via biological nitrogen fixation and phytohormone production $[1,2]$. More recently, some Herbaspirillium species have transitioned from environment (water, contaminated soil, plant internal tissues, and root nodules) to human hosts, mostly as opportunistic (pathogenic) bacteria. Cases of human colonization and infection have mostly been noted in cystic fibrosis and immunocompromised cancer patients [3-8] and also in some patients without an apparent immunosuppressed state $[9-11]$.
1.1. Case History. A 58-year-old woman with a medical history of non-small-cell lung cancer (NSCLC) with malignant left pleural effusion (with RET mutation under phase 1 trial with pralsetinib), nonbacterial thrombotic endocarditis, and pulmonary embolism presented to the hospital with fevers, chills, and rigors for 2 days. At presentation, she was febrile with a temperature of $101 \mathrm{~F}$ and tachycardic at 114 beats/minute. Physical examination revealed a Port-Acatheter on the right upper chest. Coarse breath sounds were auscultated on the right upper lung field. Labs revealed anemia with the hemoglobin level of 6.4 (ref: 12.0-16.0 g/dl), leukopenia with the white blood cell (WBC) count of 1.7 (ref 4.8-10.8 10E3/ul), mild neutropenia with the absolute neutrophil count (ANC) of 1353 cells per microliter, an elevated C-reactive protein (CRP) level of 22.52 (ref range 
$<1.00) \mathrm{mg} / \mathrm{dl}$, and an elevated creatinine level of $1.56 \mathrm{mg} / \mathrm{dl}$ (baseline creatinine of $1 \mathrm{mg} / \mathrm{dl}$ ). CT chest revealed right upper lobe pneumonia, scattered small pulmonary nodules consistent with metastatic disease, and stable bony metastatic disease of the anterior left 6 th rib. She was admitted for sepsis secondary to pneumonia and placed on broad spectrum antibiotics with intravenous piperacillin-tazobactam and vancomycin. She received 2 units of packed red cell transfusion with stable hemoglobin thereafter. Urine analysis was unremarkable. Urine legionella and streptococcus pneumonia antigens were negative.

The patient continued to have fever 2 days after admission (max: 102.8 F). Preliminary blood cultures done at our hospital at Day 2 of hospitalization revealed Gram-negative rods. CT abdomen and pelvis revealed small volume of ascites and jejunal wall thickening with mild adjacent mesenteric edema, suggestive of enteritis. CT chest revealed persistent right upper lobe pneumonia. The patient continued to have temperature spikes on the $3 \mathrm{rd}$ day of antibiotics $\left(T_{\max } 101.5 \mathrm{~F}\right.$ ). Blood cultures revealed oxidase-positive nonfermenting rods. The organism was not clearly identified in our lab, and the specimen was sent to Central Pennsylvania Alliance Laboratory for identification by DNA sequencing using MALDI (matrix-assisted laser desorption/ionization) technique. The patient's antibiotic was changed to IV meropenem on the 4th day of hospitalization. Port was removed and catheter tip culture was sent, which came out to be negative.

Ultimately, on the seventh day of hospitalization, the blood culture was identified from outside lab as Herbaspirillum seropedicae (which was susceptible to all tested antibiotics including amikacin, cefepime, ceftazidime, ciprofloxacin, aztreonam, levofloxacin, meropenem, piperacillin/tazobactam, and ticarcillin/clavulanate). The patient started feeling better and defervesced after about 24 hours of being on meropenem. The patient was discharged with a midline catheter to continue IV meropenem for a total of 14 days and follow-up for outpatient monitoring. The WBC count at discharge was $5.1 \times 10 \mu / \mathrm{L}$, ANC was 3978 cells/ microliter, and CRP was $6.36 \mathrm{mg} / \mathrm{dl}$. On further questioning to assess for risk factors, the patient reported taking care of her pond and regularly cleaning weeds.

\section{Discussion}

The first described species of the genus Herbaspirillum was the bacterium Herbaspirillum seropedicae, which was found colonizing the roots and aerial parts of important crops [1]. More recently, Herbaspirillum spp. have been recovered from the blood and sputum/bronchoalveolar lavage of patients with cystic fibrosis and pneumonia $[4,6,11]$ and from blood in patients with leukemia $[6,7,12]$, aplastic anemia [7], multiple myeloma [13], and cellulitis [8]. Our patient is a 58-year-old woman with NSCLC on RET-inhibitor pralsetinib who presented with right upper lobe pneumonia and had blood culture positive for oxidase-positive nonfermenting Gram-negative rod, which was confirmed as Herbaspirillium only on Day 7 of hospitalization by which time the patient was already improving with meropenem therapy. On retrospect, our patient had been regularly cleaning her pond and weeding her garden with possible exposure to this environmental proteobacterium, supporting this change in dynamics from environmental bacterium to opportunistic pathogen. However, it is not entirely clear why the patient only defervesced and improved after 24 hours of switching piperacillin/tazobactam to meropenem, even though her culture report suggested sensitivity to piperacillin/tazobactam. New genomic data findings suggest that the transition from environmental to pathogenic led to the loss and acquisition of specific genes to allow colonization and survival in new environments. The strains that have been infectious to humans have lost the genes for nitrogen fixation and acquired genes for lipopolysaccharide biosynthesis with the addition of sialic acids to evade the immune system [1].

We reviewed the literature for available Herbaspirillum cases and identified 9 published studies from 2005 to 2019 (Table 1), with Herbaspirillum bacteremia, cellulitis or pneumonia. Tan and Oehler reported Herbaspirillum bacteremia and cellulitis in a patient with aquatic exposure [8]. Ziga et al. reported Herbaspirillum bacteremia in a 2-yearold girl with a history of acute lymphoblastic leukemia (ALL) after induction chemotherapy and stem cell transplant [6]. Chen et al. reported Herbaspirillum bacteremia in an ALL patient on chemotherapy after taking sugarcane juice [12]. Regunath et al. described a case of bacteremia caused by gentamicin-resistant Herbaspirillum in an immunocompetent adult male farmer [11]. Suwantarat et al. reported the first fatal case-related $H$. seropedicae bacteremia secondary to pneumonia in an immunocompromised 65-year-old man with end-stage renal disease and multiple myeloma [13]. Abreu-Di Berardino et al. described Herbaspirillum huttiense pneumonia in a patient with essential thrombocytosis [9]. Liu et al. reported Herbaspirillum huttiense bacteremia in an elderly patient with no obvious immune suppression, who later went on to develop a pneumonia, where despite adequate treatment, microbiological eradication was not easily achieved, and septicemia lasted for several days along with sputum culture positivity for Herbaspirillum huttiense 2 months later [10].

Chemaly et al. investigated a potential cluster of hospitalbased Herbaspirillum infections in cancer patients at the University of Texas MD Anderson Cancer Center, initially identified as Burkholderia cepacia complex and subsequently reidentified as Herbaspirillum species by the Cystic Fibrosis Foundation Burkholderia cepacia Research Laboratory and Repository (BcRLR) at the University of Michigan. The authors identified a total of 8 patients with bacteremia and pneumonia with cultures positive for Herbaspirillum species between July 2011 and August 2012 (including 5 clusters and 3 additional cases identified prospectively). 5 of the 8 patients were females and the median age was 53 years $(2-67$ years) [7].

Spilker et al. reported a 26-year-old male with moderate to severe lung disease with Gram-negative rod bacteremia at hospital day 23. The patient was admitted for methicillinresistant staphylococcus aureus (MRSA) and Pseudomonas pneumonia, and cultures were initially misidentified as Burkholderia cepacia complex [4]. The authors analyzed 


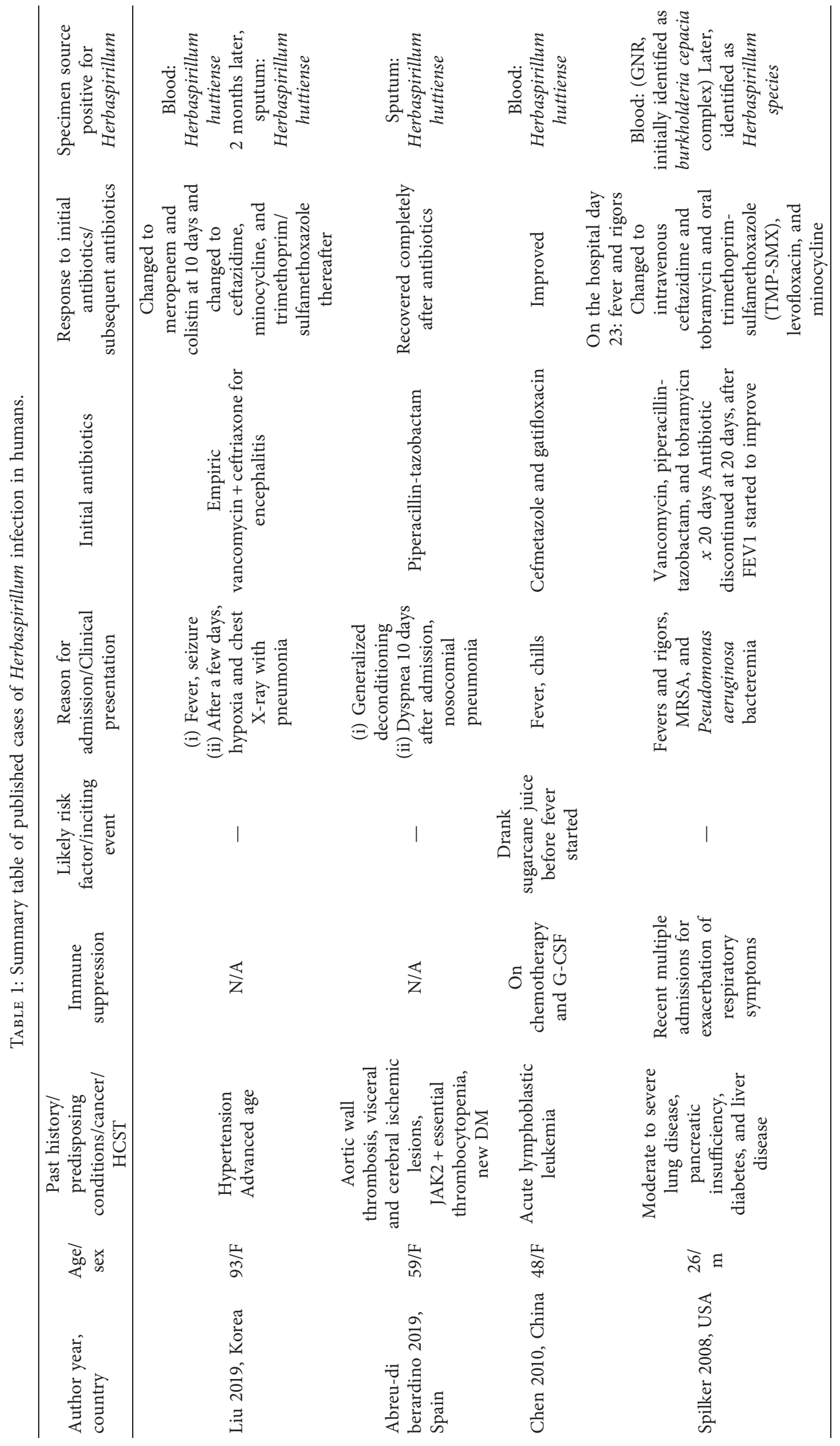




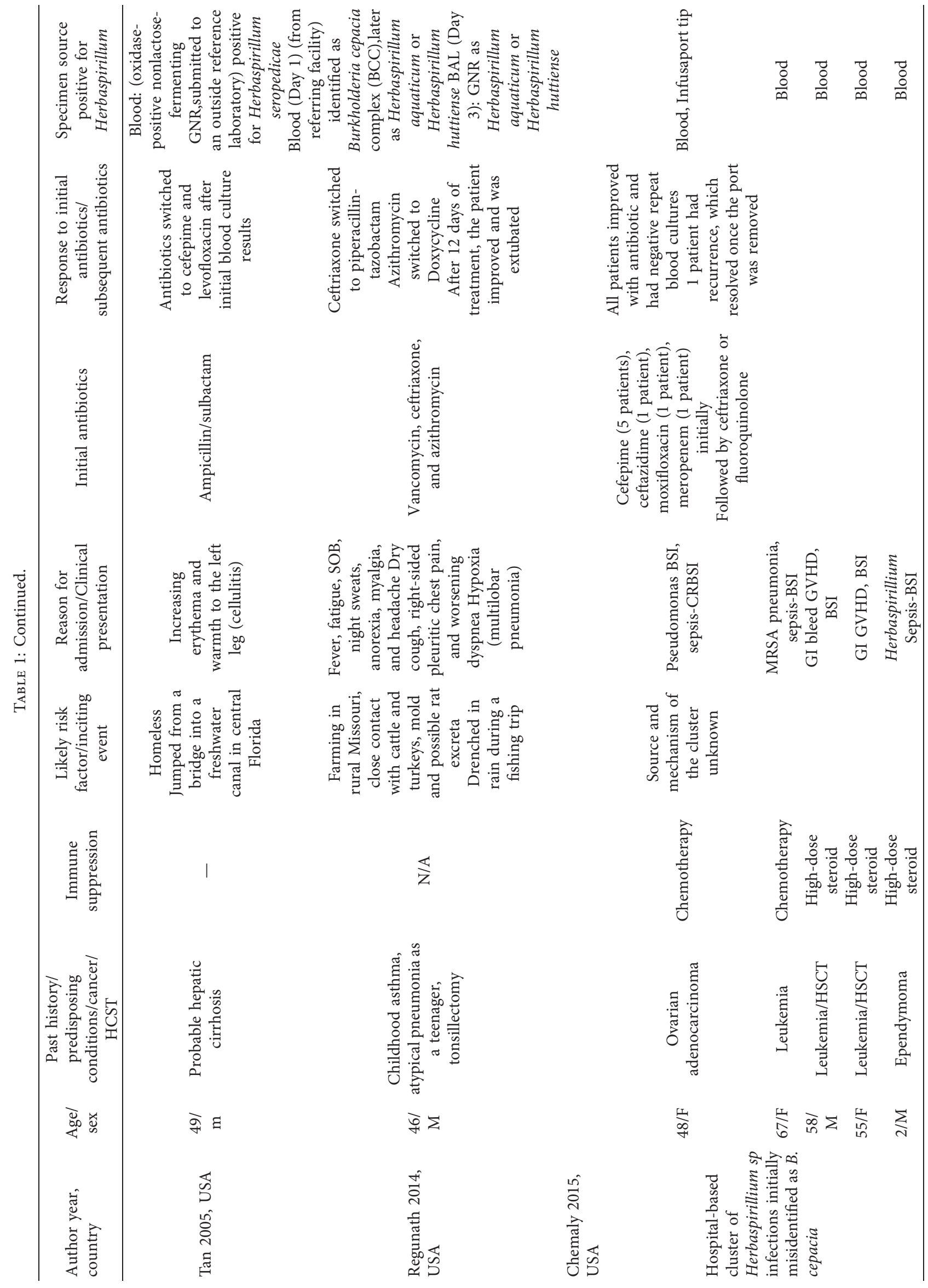




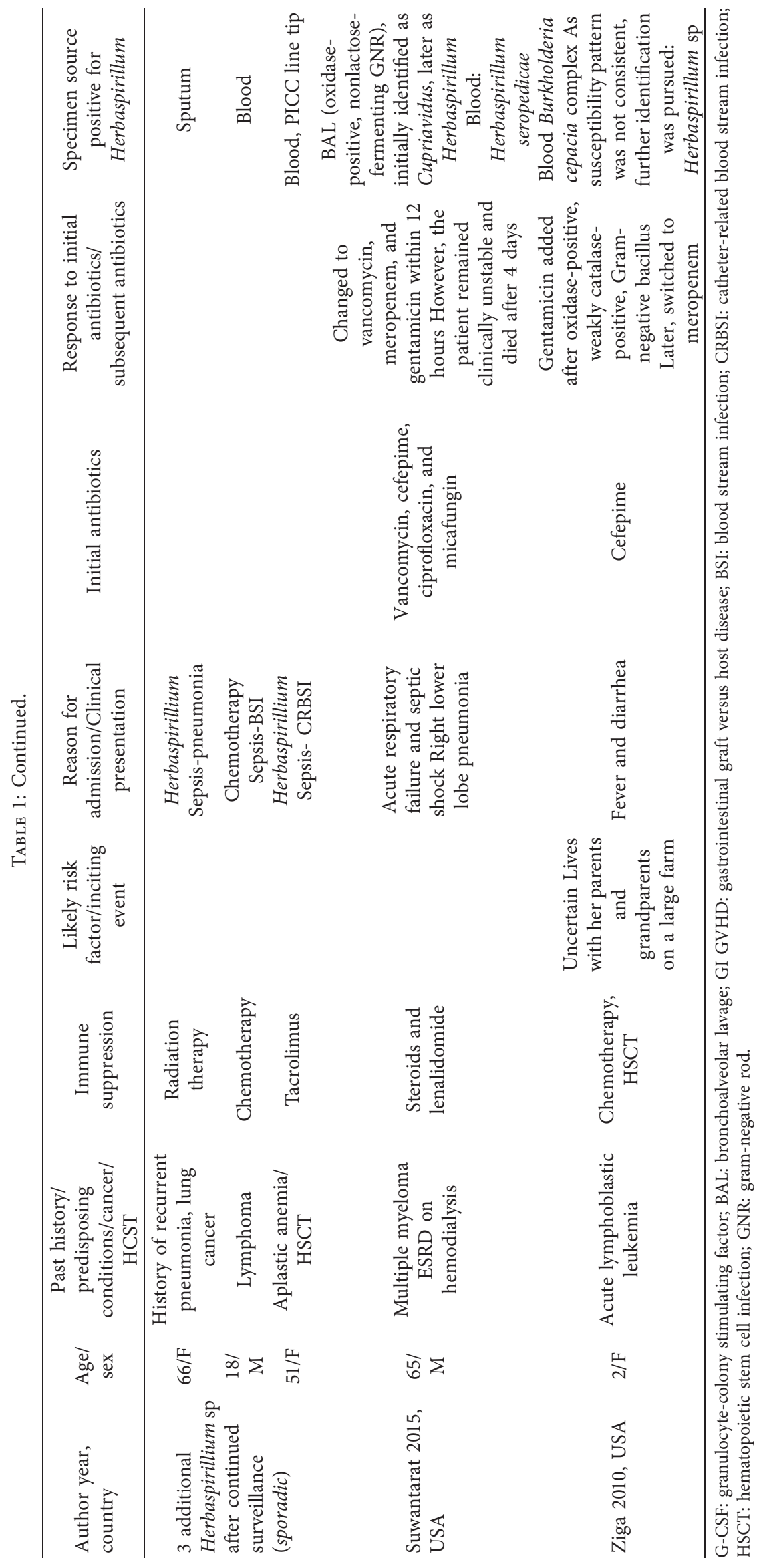


isolates from sputum from over 1,100 cystic fibrosis patients across 8 years (January 2000 to December 2007) and found Herbaspirillum in only 28 patients $(<3 \%)$ with ages ranging from 20 months to 5 years, of which 3 isolates were Herbaspirillum huttiense, 3 were Herbaspirillum frisingense, 2 were Herbaspirillum seropedicae, and 2 were Herbaspirillum putei, and the remaining eighteen isolates could not be speciated [4]. Prior to the correct identification of Herbaspirillum at the BcRLR for the above 28 specimen, they had been identified as Burkholderia cepacia complex in 19 (68\%) and Ralstonia in 4 (14\%). Most patients appeared to have had transient respiratory tract colonization with Herbaspirillum except for bacteremia in one patient and a chronic respiratory tract infection in another [4].

Tetz and Tetz, for the first time, identified Herbaspirillum frisingense from the bladder of a human patient with urinary tract infection (UTI). Furthermore, genome analysis revealed numerous factors such as adhesins, urease, hemolysin D, and pilin that contribute to the bacterium's virulence in UTIs. The authors suggested that further research of this Herbaspirillum spp would aid in better understanding of its implication in UTI [14]. Similarly, Wu et al. performed a comprehensive analysis of urinary microenvironment of bladder cancer and identified Herbaspirillum as one of the species associated with an increased risk of progression, suggesting its potential role in risk stratifying bladder cancer [15].

As evidenced by the above studies, Herbaspirillum is an emerging pathogen and may be more prevalent than earlier thought owing to misidentification for organisms like Burkholderia cepacia complex due to phylogenetic and phenotypic resemblance $[4,6,7,13]$. Antimicrobial susceptibilities may serve as a means for differentiating Herbaspirillum species from Burkholderia cepacia complex because the latter are usually multidrug resistant, whereas Herbaspirillum is not. With the institution of appropriate antimicrobial therapy, the outcomes seem mostly favorable and the bacteria appear to be easily eradicated, except in 1 case reported by Liu et al. Misidentification as Burkholderia complex can have serious implications for clinical care, and distinction between these is important due to different resistance profiles and different therapeutic implications. The increased availability of newer molecular methods (e.g., MALDI-TOF MS) should allow laboratories to correctly identify this organism and reduce misidentification by established microbial identification systems.

\section{Conflicts of Interest}

The authors declare that they have no conflicts of interest.

\section{References}

[1] H. Faoro, W. K. Oliveira, V. A. Weiss et al., "Genome comparison between clinical and environmental strains of Herbaspirillum seropedicae reveals a potential new emerging bacterium adapted to human hosts," BMC Genomics, vol. 20, no. 1, p. 630, 2019.

[2] A. C. Q. Marques, K. S. Paludo, C. B. Dallagassa et al., "Biochemical characteristics, adhesion, and cytotoxicity of environmental and clinical isolates of Herbaspirillum spp.," Journal of Clinical Microbiology, vol. 53, no. 1, pp. 302-308, 2015.

[3] T. Coenye, J. Goris, T. Spilker, P. Vandamme, and J. J. LiPuma, "Characterization of unusual bacteria isolated from respiratory secretions of cystic fibrosis patients and description of Inquilinus limosus gen. Nov., sp. Nov," Journal of Clinical Microbiology, vol. 40, no. 6, pp. 2062-2069, 2002.

[4] T. Spilker, A. Z. Uluer, F. M. Marty et al., "Recovery of Herbaspirillum species from persons with cystic fibrosis," Journal of Clinical Microbiology, vol. 46, no. 8, pp. 2774-2777, 2008.

[5] W. Chen, Y.-Y. Chen, C.-F Tsai et al., "Incidence and outcomes of acute respiratory distress syndrome," Medicine, vol. 94, no. 43, p. e1849, 2015.

[6] E. D. Ziga, T. Druley, and C.-A. D. Burnham, "Herbaspirillum species bacteremia in a pediatric oncology patient," Journal of Clinical Microbiology, vol. 48, no. 11, pp. 4320-4321, 2010.

[7] R. F. Chemaly, R. Dantes, D. P. Shah et al., "Cluster and sporadic cases of Herbaspirillum species infections in patients with cancer," Clinical Infectious Diseases, vol. 60, no. 1, pp. $48-54,2015$.

[8] M. J. Tan and R. L. Oehler, "Lower extremity cellulitis and bacteremia with Herbaspirillum seropedicae associated with aquatic exposure in a patient with cirrhosis," Infectious Diseases in Clinical Practice, vol. 13, no. 5, pp. 277-279, 2005.

[9] M. Abreu-Di Berardino, E. Rodríguez-Czaplicki, and V. Sánchez-Hellín, "Herbaspirillum huttiense pneumonia in a patient with essential thrombocythaemia," Revista Española De Quimioterapia: Publicación Oficial de la Sociedad Española de Quimioterapia, vol. 32, no. 1, pp. 83-84, 2019.

[10] C. Liu, M. J. Kwon, M. Kim, J.-H. Byun, D. Yong, and K. Lee, "Septicemia caused by Herbaspirillum huttiense secondary to pneumonia," Annals of Laboratory Medicine, vol. 39, no. 3, pp. 340-342, 2019.

[11] H. Regunath, J. Kimball, L. P. Smith, and W. Salzer, "Severe community-acquired pneumonia with bacteremia caused by Herbaspirillum aquaticum or Herbaspirillum huttiense in an immune-competent adult," Journal of Clinical Microbiology, vol. 53, no. 9, pp. 3086-3088, 2015.

[12] J. Chen, Z. Su, Y. Liu et al., "Herbaspirillum species: a potential pathogenic bacteria isolated from acute lymphoblastic leukemia patient," Current Microbiology, vol. 62, no. 1, pp. 331-333, 2011.

[13] N. Suwantarat, L. T. L. Adams, M. Romagnoli, and K. C. Carroll, "Fatal case of Herbaspirillum seropedicae bacteremia secondary to pneumonia in an end-stage renal disease patient with multiple myeloma," Diagnostic Microbiology and Infectious Disease, vol. 82, no. 4, pp. 331-333, 2015.

[14] V. Tetz and G. Tetz, "Draft genome sequence of the uropathogenic Herbaspirillum frisingense strain ureolyticus VT-1641," Genome Announcements, vol. 5, no. 17, pp. e00279-17, 2017.

[15] P. Wu, G. Zhang, J. Zhao et al., "Profiling the urinary microbiota in male patients with bladder cancer in China," Frontiers in Cellular and Infection Microbiology, vol. 8, p. 167, 2018. 